\title{
A Formal Model of Metaphor in Frame Semantics
}

\author{
Vasil Penchev $^{1}$
}

\begin{abstract}
A formal model of metaphor is introduced. It models metaphor, first, as an interaction of "frames" according to the frame semantics, and then, as a wave function in Hilbert space. The practical way for a probability distribution and a corresponding wave function to be assigned to a given metaphor in a given language is considered. A series of formal definitions is deduced from this for: "representation", "reality", "language", "ontology", etc. All are based on Hilbert space. A few statements about a quantum computer are implied: The sodefined reality is inherent and internal to it. It can report a result only "metaphorically". It will demolish transmitting the result "literally", i.e. absolutely exactly. A new and different formal definition of metaphor is introduced as a few entangled wave functions corresponding to different "signs" in different language formally defined as above. The change of frames as the change from the one to the other formal definition of metaphor is interpreted as a formal definition of thought. Four areas of cognition are unified as different but isomorphic interpretations of the mathematical model based on Hilbert space. These are: quantum mechanics, frame semantics, formal semantics by means of quantum computer, and the theory of metaphor in linguistics.
\end{abstract}

\section{INTRODUCTION}

The thesis of the paper is fourfold: (1) Metaphor can be seen as the interaction of at least two frames in a sense of frame semantics. (2) Then representation can be interpreted as the particular case of zero interaction between the frames. (3) In turn, this allows of the frames to be interpreted formally as correspondingly "reality" and the "image of reality", and language as an (even one-to-one) mapping between those two universal and formal frames of "reality" and its "image". (4) Metaphor can be further represented formally as the "entanglement" 2 of two or more frames and thus in terms of quantum information.

That thesis has advantage (or disadvantage from another viewpoint) to be self-referential and paradoxical: Indeed the sodefined concept of metaphor is in turn the interaction between two frames: both that of frame semantics and that of formal semantics and consequently it would be "only" a metaphor if the frame semantics and formal semantics can interact as this text advocates; and vice versa: if any scientific notion is expected to be a representation of reality, this text should be zero-content for

\footnotetext{
1 Dept. of Logical Systems and Models, Institute for the Study of Societies and Knowledge at the Bulgarian Academy of Sciences, Sofia, Bulgaria, email: vasildinev@gmail.com.

2 Entanglement can be interpreted as a kind of interaction due to wholeness: If two or more entities constitute a common system, they can interact with each other by the whole of the system itself, i.e. holistically, rather than only by some deterministic and unambiguous mechanism.
}

the set of its extension should be empty. Nevertheless, that explicit paradox is rather an advantage as the analogical paradox generates the development of language and thus perhaps this text as a live part of it.

The mathematical formalism of quantum mechanics (the socalled quantum mathematics) can serve for a formal theory of metaphor and thus for a serious technical formulation applicable to AI. However, the demonstration of the latter is absolutely impossible in the volume of the present paper. Its purpose is restricted only to outlining the possibility of a "quantum theory of metaphor".

That "quantum theory of metaphor" can be defined as that mathematical model of metaphor, which is based on Hilbert space very well utilized already by quantum mechanics.

Thus the suggested "quantum theory of metaphor" would share a common mathematical formalism with quantum mechanics. If that is the case, the representation of metaphor in terms of quantum mechanics is neither merely a loose analogy nor any metaphor of "metaphor", but rather a mapping between two different interpretations of the underlying model of Hilbert space.

Furthermore, the notions, approaches and even visualisations of quantum mechanics are exceptionally well developed in detail. They allow of that theory of metaphor called quantum to be represented immediately by a complete language including both mathematical model and huge practical area such as quantum mechanics.

Some of the most essential concepts of quantum mechanics necessary also to that theory of metaphor are "entanglement", "quantum information", and "quantum computer" defined bellow. Besides them, still a few terms need some specification, namely: "frame semantics", "frame" "formal semantics":

"Frame semantics" is meant in the sense of Charles J. Fillmore: "Frame semantics offers a particular way of looking at word meanings, as well as a way of characterizing principles for creating new words and phrases, for adding new meanings to words, and for assembling the meanings of elements in a text into the total meaning of the text" [1].

"Frame": "The idea is that people have in memory an inventory of schemata for structuring, classifying and interpreting experiences, and that they have various ways of accessing these schemata and various procedures for performing operations on them" [2]. "By the term 'frame' I have in mind any system of concepts related in such a way that to understand any one of them you have to understand the whole structure in which it fits ..." [1]. The "frame" already linked to formal semantics is specified as a set of well-orderings referring to something as its "logic", in which any property, relation, part or feature of that something can be understood by somebody or by some group. Consequently, that formal and semantic "frame" means the relation between the wholeness of that something and the "logic" of it as a collection of well-orderings. 
"Formal semantics" is a term used both in logic and in linguistics ${ }^{3}$ but in partially different meanings [3]. The common is the utilization of mathematical and logical models. However, the logical "formal semantics" addresses the natural entailment in language in terms of logical sequence while the linguistic "formal semantics" discusses rather the correspondence both of linguistic units and the wholeness of texts to reality in terms of mathematical mappings, set theory, and logic $[4,5]$. These meanings will be "entangled" in this paper by the mathematical concept of well-ordering, which can refer both to any logical sequence, and thus to any entailment in language, and to set theory including the axiom of choice, and thus to any one-to-one mapping of language and reality, such as a presentation.

"Entanglement" is a term in quantum mechanics, meaning the information interaction between two or more quantum systems and thus being fundamental for the theory of quantum information. However, the formal and mathematical definition of "entanglement" as that Hilbert space $^{4}$, which cannot be factorized to any tensor product of the Hilbert spaces of subsystems, allows of the term to be generalized to any model utilizing Hilbert spaces. For the formal and semantic model used here is based on Hilbert space(s), the concept of entanglement is applicable. It is the mathematical base for the model of metaphor.

"Quantum information" is a term initially coined by quantum mechanics to describe the base of a generalized kind of information underlying all quantum mechanics. So, quantum information can be interpreted as both transfinite series of bits and finite or infinite series of qubits. A bit is the elementary choice between two equally probable alternatives, and a qubit (i.e. quantum bit) can be interpreted as the elementary choice among an infinite set of alternatives though it is initially defined in quantum mechanics as the normed superposition of two orthogonal subspaces of Hilbert space. The quantity of information whether classical or quantum is the quantity of the corresponding elementary choices (whether bits or qubits) necessary for transforming a well-ordering to another (both, whether finite or transfinite). Thus quantum information can be interpreted as the quantity of elementary choices necessary to transform a frame into another and consequently the information of any metaphor formalized as above.

"Quantum computer" [7, 8, 9] is a mathematical model involved by quantum mechanics to interpret its formalism as a generalized kind of calculation, processing quantum information. Thus all physical states and processes may be also seen as computational.

The advantages of the suggested theory of metaphor would be the following:

It relies on a developed and utilized model though in a rather different scientific area.

\footnotetext{
3 Some authors doubt the relevance of formal semantics to natural languages [6].

4 The complex Hilbert space is the fundamental mathematical structure underling quantum mechanics. It is a vector space defined over the field of complex numbers. Hilbert space can be thought as the infinitely dimensional generalization of the usual three-dimensional Euclidean space where furthermore the real numbers are replaced by complex ones. Just the complex Hilbert space is meant for "Hilbert space" in the paper. It allows of: arithmetic and geometry to be generalized and thus unified into a single structure; the possible and actual to be not more than different interpretations of a single mathematical structure.
}

It can be applied practically as this is sketched (only roughly) in Section 2.

It would aid the formal reconstruction of semantic interactions as a whole as well their historical change by investigating the correlations in the uses in texts and discourses.

It allows of far reaching unifications, generalizations, and philosophical conclusions.

A section (6) is devoted to the unity of thesis as a single, coherent and contextual whole consisting of the distinguished parts (namely the four "folds" of the fourfold thesis above). The mathematical model lent by quantum mechanics is the common base.

Nevertheless some ideas can be considered in their own right even out of the model, e.g. representation as a particular, borderline and limiting case of metaphor.

However this seems to be impossible as to others, e.g. the converse relation of model and reality, proposed near the end of Section 4. Those are logical corollaries from the utilized model.

The argumentation for the thesis has four corresponding points:

(1) Metaphor can be understood as the appearance of a new frame by interaction of two or more initial frames for some essential part of each of them is shared by all. Thus the understanding of each of them separately generates immediately the understanding of the metaphor as a new whole $[10,11]$ demonstrating therefore the appearance of a new frame, which is not the simple additivity of the sub-frames composing it. The set of well-orderings formalizing semantically a frame can be substituted by a point of Hilbert space [12], and interpreted as a wave function ${ }^{5}$ of a quantum system [13]. Any possible frame is measurable as a single value of quantum information. Then the metaphor will be interpretable as the entanglement of the quantum systems corresponding to each sub-frame composing it.

(2) Representation can be interpreted after that as a particular and borderline case of metaphor, a "zero" metaphor, or just as the simple additivity of the sub-frames composing it. The corresponding wave functions are orthogonal to each other and there is no entanglement between them.

(3) Language is reduced to an infinite countable set (A) of its units of meaning, either words or propositions, or whatever others [14]. It includes all possible meanings, which can be ever expressed in the language rather than the existing till now, which would always a finite set. The external twin of reality is introduced by another set (B) such that its intersection with the above set of language to be empty. The union of them $(\mathrm{C}=\mathrm{A} \cup \mathrm{B})$ exists always so that a one-to-one mapping (f: $\mathrm{C} \leftrightarrow \mathrm{A}$ ) should exist under the condition of the axiom of choice. The mapping (f) produces an image (B (f)) of the latter set (B) within the former set (A). That image (B (f)) serves as the other twin of reality to model the reality within the language as the exact representation [15] of the reality out of language (modelled as the set B). In the model, the necessity and sufficient condition of that representation between reality both within and out of the language is just the axiom of choice: If the axiom of choice does not hold, the relation between the sets B (f) and B cannot be defined rigorously as an exact representation but rather as some

\footnotetext{
5 The term "wave function" is used bellow without quotation marks also a synonym of an element of the complex Hilbert space. Exactly speaking, the former is the common interpretation of that element in quantum mechanics.
} 
simile and the vehicle between the two twins of reality can be only metaphor ${ }^{6}$.

(4) Metaphor formalized as above is representable as the wave function of the frame compounded by two or more subframes, which interact between each other by means of the shared nonzero intersection. The quantity of quantum information of a metaphor is different from that quantity of the corresponding representation. Thus the metaphor demonstrates the entanglement of the composing sub-frames after they have been formalized as points in Hilbert space [16].

The intuitive sense for metaphor to be represented as the entanglement of its terms is the following. The meaning of any term in a metaphor influences the meanings of the rest.

Consequently, their meaning within the metaphor is essentially different from those of the terms by themselves.

Any mathematical model of metaphor needs a certain relevant quantity of that influence. Once that model involves Hilbert space(s), the entanglement and the corresponding quantity of quantum information are the most natural applicant for describing the degree of that influence.

However, the metaphor itself being already mathematically modelled serves to describe the degree of entanglement between different formal realities (or "languages") in Section 4 and Section 5. Then the formal concept of language is accordingly generalized from a simple representation of reality, i.e. its identical "twin", to a metaphorical image of both reality as a whole and its separate elements such as "things".

The paper is organized as follows. The sections from 2 to 5 argue for the four "folds" of the thesis: (1) to (4) above. Section 6 unites them into a single viewpoint. Section 7 presents the conclusions and provides directions for future work.

\section{METAPHOR AS INTERACTION OF FRAMES}

Metaphor can be seen as the interaction of two or more frames as follows. Any frame corresponds of some unit of meaning such as a word. The meaning is understood as a whole, i.e. all links between this unit and other units in the frame are actually given according to frame semantics. One can suppose language as the maximal frame containing all other frames as sub-frames. Anyway the most part of language remains absolutely or almost irrelevant to the understanding of any given term. The other, quite small part most relevant to the understanding can be used for its definition. Consequently, the understanding of a meaning can be thought as an exactly determined position in the maximal frame of language, in which the neighbour links are crucial, the next links are less crucial, and the significance of further links weaken very fast, but gradually, moving away from the position

\footnotetext{
${ }^{6}$ The axiom of choice is independent of the other axioms of set theory in the usual systems of its axioms. The former case corresponds to the systems with the axiom of choice, the latter without it. However in fact, the utilized model of Hilbert space is invariant to it without being independent of it in a sense: Quantum mechanics uses Hilbert space both with and without the axiom of choice in two interpretations of quantum mechanics, which identify to each other and anyway distinguish from each other. This is rather a special and inherent property of Hilbert space than an accidental one brought in by quantum mechanics for interpretation.
}

in question and converging to zero as to the most part of the language [17].

The same picture can be repeated for arbitrarily many meanings, and particularly for one more:

Let us figure that both meanings are simultaneously active and their joint understanding is supposed. If both meanings are neighbour or at least relevant in definition, this is rather a proposition than a metaphor. The link between them is explicit in the frame of each of them.

However that is not the case of a proper metaphor where the link connects two areas, each of which is relevant for the understanding of one term, but irrelevant for the other one.

Obviously, the transition between the compound frame of a proposition and that of a metaphor is gradual [10].

Metaphor can be seen as a generalization of proposition referring to remote meanings in the maximal frame of language. Proposition does not generate any radically new meaning irredundant to those of its parts. The meaning of a proposition can be called "analytical" in a broad and linguistic sense .

Any metaphor appeals to some implicit meaning relevant to the pathway frame between the connected ones. However, that pathway frame of a metaphor is not objective. It depends not only on the connected frame, but also on the person(s) who understand(s). The pathway and thus the implicit frame are not unambiguously determined: it includes also the personality and biography of who understands. The meaning of a metaphor can be called "synthetic" in a broad and linguistic sense:

One can utilize the picture of the maximal frame, in which are chosen two positions as two points. Furthermore, the proposition connects them by a single "classical trajectory" while, the metaphor does the same by all possible trajectories, each of which is differently probable. Any understanding chooses only one of them. The mapping analogy to the Feynman interpretation ${ }^{8}$ of quantum mechanics $[18,19,20$, and 21] is obvious. It addresses further the idea for the mathematical formalism of quantum mechanics to be only adapted to the relevant terms of frame semantics:

Indeed any measurement in quantum mechanics corresponds to a given understanding of what the metaphor mean. The metaphor unlike any proposition does not predetermine how it should be understood, however it defines implicitly a wave function of all possible understandings as the set of pathways, in any of which it can be interpreted equally justifiably.

Entanglement and the Feynman interpretation are both deduced from the mathematical formalism, but historically independent of each other. Nevertheless, there exists the following rigorous logical link between them:

The Feynman interpretation implies entanglement:

\footnotetext{
7 That "broad and linguistic sense" means that the proposition is a series, the elements of which are ordered in a whole. Anyway this is not the rigorous formal and logical deduction, which is analytical in a narrow sense for the premise implies the conclusion necessarily. The analyticity of a proposition is pragmatic and due to the possibility and probability of a rather expected link being usual and more or less often used. Metaphor is rather unexpected and nevertheless understandable.

8 The essence is any motion or change to be generalized as done in infinitely many paths simultaneously rather than in a single one. The metaphor can be thought in the same way as the motion from a term to another or others in "many paths", each of which is an interpretation of the metaphor in questions and can be realized by somebody.
} 
Indeed any "path" between two or more quantum entities means that they share at least one of their own possible states as common. And vice versa: if there is not entanglement, the Feynman interpretation would be impossible for this means that the entities are orthogonal to each other and thus they are not able to share any common states.

Furthermore, the exact mathematical formalism, which the Feynman interpretation implies, considers Hilbert space only as an approximation or as a limit after infinitely many "paths". In fact, that approximation and thus the nonzero difference between Hilbert space and the proper formalism of that kind are inherently necessary for that interpretation because this allows of entanglement to "sneak" implicitly into it.

Consequently, the Feynman interpretation is a stronger statement than the standard mathematical formulation about single, independent and thus non-entangled Hilbert spaces, which are all equivalent to a single Hilbert space ${ }^{9}$.

Once the Feynman interpretation is involved for the mathematical model of metaphor as above, this implies immediately that entanglement is also though implicitly introduced and should be discussed in the framework of that model.

The Feynman interpretation further means that if it is universal, all quantum systems are entangled, and the standard consideration of quantum mechanics by single and nonentangled Hilbert space is not more than a working idealization and simplification.

That states of affairs in quantum mechanics can be forthwith interpreted in terms of the utilized model of metaphor: Representation is not more than a working idealization and simplification of metaphor: one statement, which will be discussed in detail in the next section.

The situation of two terms can be continued to more than two, even to arbitrarily many, and one is able even to consider the case of the metaphor of metaphors [22] as well that of the "proposition of metaphors". The method for that continuation is the relevant interpretation in terms of quantum mechanics in order to be borrowed the very well developed mathematical model.

Practically, one needs some relevant, reliable, and relatively unambiguous method for any given metaphor in a given language with its use and history to be adequately determined its wave function. This method can involve the following stages:

1. Determining a broad set of associative series, which can connect the terms of the investigated metaphor.

2. Structuring this set as a directed graph [23].

3 . Determining the combinatory frequency of each vertex in the entire dictionary of the language or in any as contemporary as historical sub-dictionary if need be.

\footnotetext{
${ }^{9}$ However one has to mean that any quantum system referring to a single Hilbert space can be always exactly and equivalently represented as consisting of two or more entangled subsystems and correspondingly Hilbert spaces. Then the viewpoint of the system differs from that of any subsystem. The Feynman interpretation is a way the viewpoint of the quantum whole to be represented as a certain function (namely its wave function) of the viewpoints of its virtual classical "parts", each of which is featured by a single classical "path". The suggested model of metaphor being considered as a whole would consist of the virtual parts of its interpretations, any of which is featured by its own proper associative path and a corresponding probability of this path calculable by relevant frequency uses.
}

4. Calculating the frequency and probability in any possible pathway in the graph.

5. Summarizing these data as a probability distribution.

6. Approximating this probability distribution [24] by a wave function.

7. Eventually interpreting and modelling this wave function as a state of a quantum system and thus of a quantum computer.

Only stage 1 depends crucially on the human creativity to be figured all thinkable and unthinkable associative series, which can connect the terms of a metaphor. All rest stages can be accommodated for relevant software.

However, ever this first stage might be replaced by a formal frequency use analysis of common terms in the frames of all terms constituting a given metaphor. One should consider those frames as frequency use in the context of a given term and consisting of two, three, four and so on words. Consequently, the following stages « 1 '» and « 1 "» can substitute the above « 1 »:

1 ': Formally determining the frame of each term constituting the given metaphor as frequency uses of two, three, four, five, and so on words, containing the term in question.

1": Determining the frequency use of common terms in the frames of the terms of the investigated metaphor.

Those stages can be quite roughly illustrated by an imaginary example for their application about a real metaphor, e.g. "The moon is sad".

First of all, this is an obvious metaphor, which connects a celestial body, which is impossible to be sad, with a human mood, that to be sad: Who is sad cannot be anything inanimate such as the moon.

Furthermore, "Google" shows that the exact phrase as above is used in 59,000 web sources (retrieved on 14.03.2015). Nevertheless, the phrase is found in no case in the huge data base of English literature in "Ngram Viewer" of "Google books" (again then). Consequently, this is a real contemporary metaphor rather than a "white metaphor" coining Derrida's metaphor about any too used metaphor.

There are at least two different practical methods, which would give also different results perhaps, to be determined the paths and their corresponding probabilities for the latter term, "sad", to be reached starting from the former term, "moon".

The one method would construct the frames of both terms by means of main frequency uses of small contexts containing the terms and would search for coincidences of terms belonging to both frames.

One can figure as an imaginary example that the pair (moon, round) has frequency use " $f_{1}$ " and probability " $p_{1}$ " calculable as the ratio of " $\mathrm{f}_{1}$ " to the number of all considered frequency uses in the frame of "moon". Furthermore, the triple (round, face, sad) is analogically featured by " $\mathrm{f}_{2}$ " and " $\mathrm{p}_{2}$ " in the frame of "sad". "Round" is the searched coincidence. It allows of constructing some relevant function " $\mathrm{P}_{1}\left(\mathrm{f}_{1}, \mathrm{p}_{1}, \mathrm{f}_{2}, \mathrm{p}_{2}\right)$ ", which would suggest a value of the composed path (moon, round, face, sad) connecting both terms of the metaphor in a possible way.

The other method would consider only the frequency uses of those pairs, the series of which starts from "moon" and finish to "sad".

In the above example, those would be: (moon, round), $\mathrm{f}_{3}, \mathrm{p}_{3}$; (round, face), $\mathrm{f}_{4}, \mathrm{p}_{4}$; (face, sad), $\mathrm{f}_{5}, \mathrm{p}_{5}$. They would imply some $\mathrm{P}_{2}\left(\mathrm{f}_{3}, \mathrm{p}_{3}, \mathrm{f}_{4}, \mathrm{p}_{4}, \mathrm{f}_{5}, \mathrm{p}_{5}\right)$ of the same path however calculated by the latter method. 
If that procedure either in the former or in the latter method is repeated as to many enough paths, one can yield the probability distribution, which refers to the metaphor "The moon is sad" in English, with any preliminarily defined exactness. Then, the characteristic function of that probability distribution will represent the searched wave function of the metaphor in question.

The above two methods can be further modified and mixed in different proportions. However, they reflect two different ways for the model of metaphor to be understood: either as the entanglement of the frames of terms constituting a given metaphor or as a single frame of the metaphor as a whole, which is practically reduced to a set of series corresponding to paths between the terms of the metaphor.

Anyway the goal of the paper is only the possibility in principle as well as a schematic diagram of how the metaphors first interpreted in terms of frame semantics to be further modelled mathematically and then computationally.

\section{REPRESENTATION AS A PARTICULAR CASE OF METAPHOR}

The next step refers to representation: How the representation to be grounded on metaphor? The usual way is the reversed: How the metaphor to be founded by representation, which is granted as a self-obvious base?

However, the above mapping to quantum mechanics leads just to the metaphor to be the starting point. The end point is not so the representation by itself, but the concept of reality to be obtained in a formal and mathematical way [25] in order to be modeled.

The representation can be considered as a particular and borderline case of metaphor following the method for quantum mechanics to be reduced to classical mechanics by the principle of correspondence.

The problem is the following. Some metaphor is given. Which are the boundary conditions, on which its wave function can be transformed into that of a corresponding representation? The wave function of a representation is degenerated in a way so that the corresponding probability distribution is reduced to a single infinite pick in a single point, i.e. to the Dirac $\delta$-function.

That result for the probability distribution in all associative ways of the metaphor in question can be obtained so: the interval of nonzero probabilities converges to the limit of a single point

The process of convergence requires both decreasing the associative "distance" between the connected terms of the metaphor (which are at least two) and increasing the extension of the generalization of the terms so that the set of all associative pathways to be able to be reduced gradually to a single one. If that is the convergence, the corresponding directed graph of the metaphor will degenerate to a directed segment and even to a directed segment of zero length. The latter in turn is equivalent to a bit of information [26]: the "cell" of the segment possesses two equally probable, but alternative state of each of the two ends.

This would correspond to the degenerated or "ontological" metaphor: " $\mathrm{A}$ " is $\mathrm{A}$ ' decodable as the dialectic judgment that both " $\mathrm{A}$ " is $\mathrm{A}$, and " $\mathrm{A}$ " is not $\mathrm{A}$. The two ends of the "zero segment" are: "A" and A (whatever A is).

The directed segment of zero length (or a bit) means an elementary choice as well as an identical mapping. If these concepts are applied to an infinite set, they require the axiom of choice and even a special case of invariance in relation to it. That invariance consists in this, any subset of any set not only to be able to be enumerated by virtue of the axiom of choice, but also the set and the enumerated image of it to be identified.

The mathematical model of representation deduced from the metaphor should include all aforesaid formal properties.

Let us now interpret these mathematical features of representation in terms of frame semantics, i.e. as an interaction between two frames, which relation can be even identical. That interaction is zero in both opposite cases: both where the frames are absolutely independent of each other and where they coincide.

Even more, both cases can be identified by the above formal properties of representation as the "two ends of a directed segment of zero length" or as the "ontological metaphor": "A" is $A^{\prime}$.

Then the "class of all representations" can be defined as 'reality' in terms of the formal frame semantics. Reality can be deduced from representation, which in turn can be deduced from metaphor.

The formal and mathematical concept of reality is crucial for modeling any intellect able to be standalone. The demarcation line between a machine however "clever" and an intellect however "stupid" is just the concept of reality, which is inherent for the latter and somebody else's for the former. Thus the machine however "intelligent" remains a machine in somebody else's reality, e.g. a human being's.

Reality equivalent to the class of all representations is equivalent also to the aforesaid invariance to the axiom of choice for the class of all representations coincides with that invariance. However, it can be defined only on infinite sets.

Practically, this means that the formal concept of reality defined as above can be modeled only by some quantum system, i.e. on a quantum computer rather than on a Turing machine (i.e. on any standard computer independent of its power) always representing always a finite series after finishing effectively by any result

A representation modeled on a quantum computer is a measurement of it. Any direct measurement means for a quantum computer to be irreversibly demolished, though:

This means that the superposition of all possible states, which is essential for its definition, is reduced to a single one, namely what is measured. Indeed the processing of a quantum computer consists in a reversible and smooth change of all elements of a set of probability distributions. Thus the statistical probabilities of the corresponding ensemble of measured results are changed as the output of that computer. However, the measurement of any state cancels irreversibly its work and it is destroyed in fact.

Consequently, the attempt to be modeled that formal concept of reality on a quantum computer fails for the set of representations, i.e. measurements are not infinite: even if the measurements are done of a collection of quantum computers. Furthermore, that collection is not only finite, but also a statistical ensemble rather than a coherent state.

One has to search for other, nondestructive ways for mappings of a coherent state into another or other of a quantum computer rather than into the elements of a statistical ensemble.

This requires the correspondence of reality and image to be first reformulated in a generalizing way allowing of the communication between them by means of entanglement. 


\section{HILBERT SPACE: REALITY AND ITS MAPPING WITHIN A QUANTUM COMPUTER}

The next step refers to the formal concept of language again by means of Hilbert space [27, 28]. The goal of that step addresses reality to be generalized in way allowing of sharing reality not to lead to demolishing the quantum computer. The constraints and quantitative laws of that sharing are further problems.

Once reality is defined formally as a special set of mappings, one can continue generalizing to broader and broader sets of mappings. They can be also considered as "languages" mapping the so defined "reality" in different ways. Furthermore, each that language offers a different metaphor in general ${ }^{10}$ for each "element of reality" being a representation. Then any collection of metaphors about those "elements of reality" is a language obviously defined already formally.

In other words, the language is defined as a particular set of primary (or "elementary") metaphors, in which at least one term is necessarily an "element of reality" while the others designate or define it. Two frames correspond to them in frame semantics being linked to each other by a wave function, i.e. by a point in Hilbert space according to the model introduced in section 2 .

This means that any language should be consider as a state of the quantum field over reality. The term of "quantum field" is meant as usual in quantum mechanics, i.e. as a mapping of a set (the set of all representations, or "reality") into Hilbert space.

The "set of all possible states of the so-defined quantum field" including all possible languages will be designate as 'ontology',11.

Consequently, the concept of ontology is implied much broader than that of reality. If any image of reality in any language is interpreted as another reality, then ontology is the class of all realities or of all possible worlds.

One can demonstrate that those formal concepts are able to be modelled entirely within Hilbert space in a quite natural way. Indeed "representation" corresponds to the relation of two coinciding elements of the two dual spaces. They are both identical and complementary.

Consequently, the so-defined formal concept of reality is inherent to Hilbert space. If Hilbert space is considered as a model shared e.g. by quantum mechanics, that reality is internal rather than external to it. It is complete to that reality.

The interrelation of model and reality (more exactly, the sodefined reality as a formal model) is rather extraordinary in comparison with classical physics, science, and epistemology, being "reversed" in a sense. Model contents the model of reality rather than reality contents the reality of model.

Then any language is a mapping of Hilbert space [29] into itself, and thus any physical quantity ${ }^{12}$ is a language defined formally as above (but not vice versa).

Furthermore, Hilbert space can be considered as a quantum computer, and any point in it as a state of it. So that quantum computer should content reality in the sense of the above formal model of reality within itself being therefore standalone rather than a machine within somebody else's reality.

\footnotetext{
${ }^{10}$ Particularly some metaphors in some languages can coincide.

${ }^{11} \mathrm{~T}$. Giraud offers a fundamentally different ontological perspective [32].

${ }^{12}$ In the way as it is defined in quantum mechanics.
}

However, there is a considerable problem of how two or more different realities are able to communicate. Particularly, how is a quantum computer able to transfer a result to us without demolishing itself and thus destroying also that other reality within it and different from ours?

As we will see: only "metaphorically".

\section{METAPHOR IN TERMS OF ENTANGLEMENT}

The next step requires the relation of any two "languages" to be defined in terms of Hilbert space(s) therefore involving entanglement between them. The goal is: some nondestructive way for transmitting information between two or more realities identified as languages to be outlined. The way of measurement has already excluded above as destructive.

Let there are two different "metaphors" of one and the same "element of reality" in two languages, i.e. two wave functions. The "element of reality" can be excluded and any of the two metaphors can be directly referred to the language (reality) of the other. Those language and reality in the neighborhood of the metaphor are unambiguously defined by the corresponding wave function. Thus the metaphor will "seem" or "appear" as the entanglement of both wave functions from the viewpoint of each of the languages.

One can compare the formal definition of a metaphor in Section 2 as a single wave function with the present definition as the entanglement of two ones. Obviously, these definitions do not coincide: There are two different definitions of one and the same metaphor therefore each one needing some different, but relevant interpretation:

The metaphor defined as in Section 2 as a single wave function should be interpreted as that in the common system of the language or in the universal reality to the particular realities of each term.

The metaphor defined as here, in Section 5 as the entanglement of two or more wave functions should be interpreted as seen from the particular viewpoint of each term of it and thus in the corresponding particular reality.

However, that mismatch is just the nondestructive way for a quantum computer to transmit a result, as we see, only "metaphorically". The transfer is "less metaphorical", i.e. more precious, the quantum computer will be more influenced by the transfer, even demolished after any absolutely exact transmission of its result. The mismatch depends on the quantity of entanglement, in particular, on that of the quantum computer and our reality.

If one of the terms of the metaphor is permanent, e.g. anchored in our reality, the change of the others can be interpreted as the metaphorical "message" thus poetically [30, 31]. The quantum computer turns out to be a "poet".

Practically, the transmitted result will be a change of the rest frames to an anchored frame postulated as that of reality as to our reality. That change of a few frames being also a change of metaphor and an arbitrary ${ }^{13}$ operator in Hilbert space can be defined as a single elementary thought [33].

Consequently, a quantum computer cannot report a result in a nondestructive way, but can communicate a thought just as a

\footnotetext{
${ }^{13}$ That is neither self-adjoint, nor linear in general.
} 
human being can. If the thought is clearer, the computer is more "obsessed" by it: i.e. its state and thus future work will be more influenced by its communication.

\section{THE UNITY OF THESIS}

One can deduce the following from summarizing Sections $2-5$ :

From 2: Metaphor can be represented as an interaction of frames in terms of frame semantics, and then modelled formal as a "wave function", i.e. as an element (point, vector) in Hilbert space.

From 3: Representation can be defined as a particular case of metaphor, namely as the directed segment between two coinciding frames with a corresponding probability distribution degenerated to a Dirac $\delta$-function. The set of all representations is a formal definition of reality.

From 4: That reality turns out to be inherent and internal to Hilbert space and thus to any quantum computer. It can be also considered as identical to a formal concept of language. The class of all languages (or "realities") defines formally the concept of ontology.

From 5: A quantum computer can report a result only "metaphorically" or "poetically". The report is more precise, the quantum computer is more influenced; and even demolished in the borderline case of absolutely exact report. That report is a change of a metaphor to an anchored term and can be considered as a formal definition of thought.

Conclusion from 2 to 5: Any quantum computer being furthermore standalone and supplied by reality can think. Human thinking can be exhaustedly modelled by a quantum computer.

The unity of the thesis includes a few heterogeneous fields of cognition: quantum mechanics as a theory of nature, frame semantics as a theory of human thinking, the theory of metaphor and representation as a theory of language, quantum computer as a theory of artificial intellect. The four can share a common mathematical model based on Hilbert space(s). This allows of a uniform and even mathematical description both of thinking whether human or artificial and of states and process whether physical or linguistic. These four can be considered as not more than different interpretations of a single model and thus isomorphic to each other.

\section{CONCLUSIONS \& FUTURE WORK}

This paper shows how one can use the concept of frame in frame semantics to define metaphor as an interaction of frames. The Feynman "many-paths" interpretation of quantum mechanics allows of the metaphor to be represented by a wave function and thus the mathematical model of Hilbert space to be involved.

One can demonstrate a general approach for any given metaphor in any given language to be assigned a relevant probability distribution and then a wave function. Though the approach is shown by the example of two terms, it can immediately extend to more than two terms following the pattern of quantum mechanics: any separate position in the Feynman model corresponds one-to-one with a term of the metaphor.

The formal model of metaphor implies that of representation as a particular and borderline case of the "ontological" metaphor " $A$ " is A', and the Dirac $\delta$-function as the corresponding probability distribution. This allows of a formal definition of reality as the set of all representations. That reality is inherent and internal to Hilbert space. Thus any quantum computer turns out to be supplied by its inherent and internal reality. Its reality is what guarantees for it to be standalone rather than a machine in somebody else's reality. However, a quantum computer cannot report us any absolutely exact result without self-demolition.

One can define a formal concept of language within Hilbert space as the mapping of "reality", being internal to the Hilbert space, to the same Hilbert space. That mapping can be considered as a quantum field in the standard definition of quantum mechanics. However, it can be also interpreted as a language mapping any element of reality (signified) into another (signifier) by means of that metaphor (sign), the wave function of which is the value of the quantum field for this element of reality. Furthermore one can define "ontology" as the "class of all languages" and therefore of all realities or all possible worlds.

This allows of another formal definition of metaphor as a compound "sign" (i.e. two or more entangled wave functions) consisting of two or more signs referring to different signifier in different languages, but of a single common signified.

That formal concept of language is a "quantum field" on "reality", i.e. as a mapping of the set of the formally defined reality in Hilbert space into the same Hilbert space. Any "element of reality" is a "signified" mapped by the "sign" of a metaphor (i.e. a wave function) into another (in general) "element of reality" as a "signifier". Any "language" is also interpreted as another and different "reality" again formally defined. 'Ontology' is further defined as the "class of all languages" and thus that of all realities.

The other, new, and different formal definition of metaphor is given as the relation between different signifiers of a single element of reality as a signified and therefore modeled by two or more entangled wave functions corresponding to the sign of each term in each language.

There will be two distinct definitions of one and the same metaphor: as a single wave function according to Section 2 and as a few entangled wave functions according to Section 5 . The quantitative mismatch (being due to the entanglement) between the two definitions can be represented back in terms of frame semantics as a change of a frame to another, after which all rest terms will change their position to one anchored to that reality (language) chosen as a reference frame, e.g. ours.

That "frame change" being also a "metaphor change" can be defined as an 'elementary thought' [34].

Any quantum computer can transmit any result in a nondestructive way only "metaphorically" or "poetically" rather than literally, i.e. as an elementary thought. The thought transmits the result more exact, it is more "obsessive" for the computer: that is its state and thus reality is more influenced by the event of transmission. The borderline case of an absolutely exact report of the result is tantamount to its demolition.

One can also say that quantum computer thinks in this sense of transferring a message between realities (or languages) metaphorically. Furthermore, the essence of thought turns out to metaphorical and thus poetical in the frame of the present paper.

The unity of the thesis demonstrates that a single and common mathematical model based on Hilbert space can be shared by four scientific fields: quantum mechanics describing nature; frame semantics describing human cognition; linguistics 
describing metaphor and representation; theory of quantum information describing quantum computer.

That unity implies the following five directions for future work. Four ones for each of the four fields enumerated above and still one, the fifth for their synthesis developing the underlying mathematical model.

\section{REFERENCES}

[1] C. J. Fillmore. Frame semantics. In: Linguistics in the Morning Calm. Linguistic Society of Korea (Ed.) Hanshin, Soeul, Korea: 111-137 (1982).

[2] C. J. Fillmore. Frame semantics and the nature of language. In: Origins and Evolution of Language and Speech. S. R. Harnad, H. D. Steklis, J. B. Lancaster (Eds.) (Annals of the NY Academy of Sciences, Vol. 280) New York Academy of Sciences, New York, USA: 20-32 (1976).

[3] J. Woleński. What is Formal in Formal Semantics? Dialectica, 58(3): 427-436 (2004).

[4] J. Woleñski. From Intentionality To Formal Semantics (From Twardowski To Tarski). Erkenntnis , 56(1): 9-27 (2002).

[5] R. Rogers. A survey of formal semantics. Synthese, 25 (1): 17-56 (1963).

[6] A. Galton. Formal semantics: is it relevant to artificial intelligence? Artificial Intelligence Review, 2(3): 151-165 (1988).

[7] R. P. Feynman. Simulating Physics with Computers. International Journal of Theoretical Physics, 21(6/7): 467-488 (1982).

[8] R. P. Feynman. Quantum Mechanical Computers. Foundations of Physics, 16(6): 507-531(1986).

[9] D. Deutsch. Quantum Theory, the Church-Turing Principle and the Universal Quantum Computer. Proceedings of the Royal Society of London. Series A, Mathematical and Physical Sciences, 400 (1818): 97-117 (1985).

[10] L. J. Cohen. The semantics of metaphor. In: Knowledge and Language: Selected Essays of L. Jonathan Cohen. J. Logue (Ed.) Dordrecht, Springer Netherlands: 27-40 (2002).

[11] F. Guenthner. On the semantics of metaphor. Poetics, 4(2-3): 199 220 (1975).

[12] A. Y. Khrennikov. Ubiquitous Quantum Structure. Springer, Berlin; Heidelberg (2010).

[13] D. Aerts and M. Czacho. Quantum aspects of semantic analysis and symbolic artificial intelligence. Journal of Physics A: Mathematical and General, 37 (12): 123-132.

[14] M. Jubien. Formal Semantics and the Existence of Sets. Nô̂s, 15(2): 165-176 (1981).

[15] J. H. Dreher. On the semantics of formal representation. Philosophia: Philosophical quarterly of Israel, 8(1): 71-78 (1978).

[16] D. Aerts and L. Gabora. A theory of concepts and their combinations I: The structure of the sets of contexts and properties. Kybernetes, 34(1\&2): 151-175 (2005).

[17] C. Gauker. Contexts in Formal Semantics. Philosophy Compass 5(7): 568-578 (2010).

[18] R. P. Feynman. Space-Time Approach to Non-Relativistic

Quantum Mechanics. Reviews of Modern Physics, 20(2): 367-387 (1948).

[19] R. P. Feynman and F. L. Vernon, Jr. The theory of a general quantum system interacting with a linear dissipative system. Annals of Physics (N.Y), 24: 118-173 (1963).

[20] R. P. Feynman; A. R. Hibbs. Quantum mechanics and path integrals. New York: McGraw-Hill (1965).

[21] H. D. Zeh. Feynman's interpretation of quantum theory. The European Physical Journal H, 36(1): 63-74 (2011).

[22] M. Bergmann. Metaphor and formal semantic theory. Poetics, 8(12): 213-230 (1979).

[23] W. J. Hutchins. Semantics in three formal models of language. Lingua, 28(1): 201-236 (1972).
[24] P. Bruza, J. Busemeyer , and L. Gabora. Introduction to the special issue on quantum cognition. Journal of Mathematical Psychology, 53: 303-305 (2009).

[25] D. Pearce and V. Rantala. Realism and formal semantics. Synthese, 52(1): 39-53 (1982).

[26] L. V. Arshinskii. Substantial and Formal Deductions in Logics with Vector Semantics. Automation and Remote Control, 68(1): 139-148 (2007).

[27] A. Khrennivov. Classical and Quantum Mechanics on Information Spaces with Applications to Cognitive, Psychological, Social, and Anomalous Phenomena. Foundations of Physics, 29(7): 1065-1098 (1999).

[28] D. Aerts and L. Gabora. A state-context-property model of concepts and their combinations II: A Hilbert space representation. Kybernetes, 34(1): 192-221 (2005).

[29] C. Begz. Mathematical Approaches to Cognitive Linguistics. International Journal of Applied Linguistics \& English Literature, 2(4): 192-199 (2013).

[30] T. Van Dijk. Formal semantics of metaphorical discourse. Poetics, 4(2-3): 173-198 (1975)

[31] E. Bruss. Formal semantics and poetic meaning. Poetics, 4(14-15): 339-363 (1975).

[32] T. Giraud. Constructing formal semantics from an ontological perspective. The case of second-order logics. Synthese, 191(10): 2115-2145 (2014).

[33] N. Chino. Hilbert space theory in psychology (1) - Basic concepts and possible applications. Bulletin of the Faculty of Letters of Aichi Gakuin University, 28: 45-65 (1998).

[34] A. Ortony. Metaphor, language, and thought. In: Metaphor and thought. A. Ortony (Ed.) Cambridge University Press, Cambridge; New York: 1-16 (1979). 


\section{1st Annual Convention of the}

Society for the Study of Artificial Intelligence and the Simulation of Behaviour

\section{(AISB 2015)}

Canterbury, UK

20-22 April 2015 
Printed from e-media with permission by:

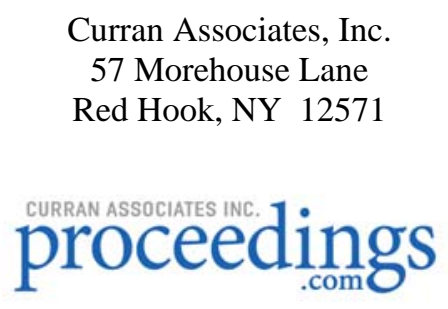

Some format issues inherent in the e-media version may also appear in this print version.

Copyright $@$ (2015) by AISB - The Society for the Study of Artificial Intelligence and the Simulation of Behaviour

All rights reserved.

Printed by Curran Associates, Inc. (2015)

For permission requests, please contact AISB

at the address below.

AISB

c/o Dr. Katerina Koutsantoni

4 Windsor Walk, Denmark Hill

London SE5 8AF UK

Phone: 441273678448

Fax: $\quad 441273671320$

aisb@cogs.susx.ac.uk

\section{Additional copies of this publication are available from:}

Curran Associates, Inc.

57 Morehouse Lane

Red Hook, NY 12571 USA

Phone: 845-758-0400

Fax: $845-758-2634$

Email: curran@proceedings.com

Web: www.proceedings.com 


\section{TABLE OF CONTENTS}

\section{AI AND GAMES}

Modelling Cultural, Religious and Political Affiliation in Artificial Intelligence Decision-Making Mark R. Johnson

Discerning Human and Procedurally Crafted Content for Video Games Tommy Thompson, Rob Watling

Hybrid Procedural Content Generation: A Proposal Michael Cook, Simon Colton

Revealing Social Identity Phenomena in Videogames with Archetypal Analysis Chong-U Lim, D. Fox Harrell

PALAIS: A 3D Simulation Environment for Artificial Intelligence in Games 18 Patrick Schwab, Helmut Hlavacs

Simulating Autonomous Non-Player Characters in a Capture the Flag Scenario Using PALAIS ............................22 Patrick Schwab, Helmut Hlavacs

EmohawkVille: Virtual City for Everyone David Holan, Jakub Gemrot, Martin Cerny, Cyril Brom

An Interactive, Generative Punch and Judy Show Using Institutions, ASP and Emotional Agents Matt Thompson, Julian Padget, Steve Battle

Search and Recall for RTS Tactical Scenarios Jason Traish, James Tulip, Wayne Moore

Follow-up on Automatic Story Clustering for Interactive Narrative Authoring Michal Bída, Martin Cerny, Cyril Brom

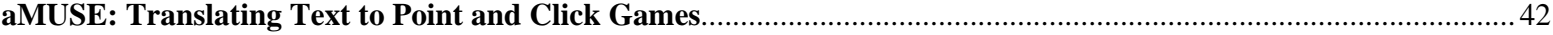
Martin Cerny, Marie-Francine Moens

Data Collection with Screen Capture. Jason Traish, James Tulip, Wayne Moore

Cognitive Navigation in PRESTO Paolo Calanca, Paolo Busetta

\section{UPDATING THE ANTI-REPRESENTATION DEBATE: BEHAVIOR-ORIENTED PERSPECTIVES}

Introduction N/A

Information and Dynamics in Brain-Body-Environment Systems 55 Randall D. Beer

Perception in Action: Radicality in Cognition and How to Resist it. 56 Gabriele Ferretti

Growing Minds from a Different Seed: How Focusing on the Basis of Behavior Induces a Radically

Different Theory of Cognition Martin Flament Fultot

The Behavior-based Origin of Life and the Problem of Genetic Representation Tom Froese

A Dynamical Multi-scaled Approach to Sensorimotor Behavior Raoul Huys

Agents and Organisms: Why the Difference is Important for the Representation Discussion (and

Cognitive Science in General) Fred Keijzer

Adaptive Behavior Through Synchronization and Compliance Brian Mirletz

Finding Home Without Knowing Where You Are: Visually Guided Navigation Without Mapping Or

Object Recognition Andrew Philippides

Moving Beyond On- and Offline Cognition.

Ludger van Dijk, Rob Withagen 


\section{SOCIAL ASPECTS OF COMPUTING AND COGNITION}

Introduction

Raffaela Giovagnoli, Gordana Dodig-Crnkovic

The Enactive Theory of Social Cognition: From Theory to Experiment Tom Froese

The Dual Sociality of Big Data Practices: Epistemological, Ethical and Political Considerations. 70 Judith Simon

Reasoning In Mathematics and Machines: The Place of Mathematical Logic in Mathematical

Understanding...... Danielle Macbeth

Propagation of the Effects of Certain Types of Military Psychological Operations in a Networked Colette Faucher

Projective Simulation and the Taxonomy of Agency.....

Leon Homeyer, Giacomo Lini

Rationality in the Behaviour of Slime Moulds and the Individual-Collective Duality Andrew Schumann

Reasoning, Representation and Social Practice (Extended Abstract) Rodger Kibble

The Search for Computational Intelligence. Joseph Corneli, Ewen Maclean

Digital Footprints: Envisaging and Analysing Online Behaviour. Giles Oatley, Tom Crick, Mohamed Mostafa

On the Rationality of Emotion: A Dual-system Architecture Applied to a Social Game David C. Moffat

Collective Cognition and Distributed Information Processing from Bacteria to Humans Alexander Almér, Gordana Dodig-Crnkovic, Rickard von Haugwitz

Social Computing Privacy and Online Relationships Gaurav Misra, Jose M. Such

Computational Aspects of Autonomous Discursive Practices Raffaela Giovagnoli

Digital Identity: Finding Me. Yasemin J. Erden

8TH AISB SYMPOSIUM ON COMPUTING AND PHILOSOPHY: THE SIGNIFICANCE OF METAPHOR AND OTHER FIGURATIVE MODES OF EXPRESSION AND THOUGHT

Introduction John Barnden, Andrew Gargett

Metaphor, Fiction and Thought John Barnden

Metaphor and Understanding $M e$ Yasemin J. Erden

Automatic Metaphor-Interpretation in the Framework of Structural Semantics 154 Christian J. Feldbacher

Metaphorical Minds, Illusory Introspection, and Two Kinds of Analogical Reasoning...... Eugen Fischer

Metaphors in Theory of Information: Why They Capture Our Concepts and Undertakings Marek Hetmanski

From Metaphor to Hypertext: An Interplay of Organic and Mechanical Metaphorics in the Context of New Media Discovering ..... Zuzana Kobíková, Jakub Mácha

Metaphor, Meaning, Computers and Consciousness Stephen McGregor, Matthew Purver, Geraint Wiggins

A Formal Model of Metaphor in Frame Semantics Vasil Penchev

How Can Metaphors Be Interpreted Cross-linguistically? Yorick Wilks

Relevance Theoretic Comprehension Procedures: Accounting for Metaphor and Malapropism .202 Zsófia Zvolenszky 


\section{COMPUTATIONAL CREATIVITY}

The Creativity of Computers at Play David C. Moffat

An Informational Model for Cellular Automata Aesthetic Measure .... Mohammad Ali Javaheri Javid, Mohammad Majid al-Rifaie, Robert Zimmer

Tightening the Constraints on Form and Content for an Existing Computer Poet 220 Pablo Gervas

Four PPPPerspectives on Computational Creativity Anna Jordanous

Towards a Computational Theory of Epistemic Creativity Jir Wiedermann, Jan van Leeuwen

How Many Robots Does It Take? Creativity, Robots and Multi-Agent Systems 243 Stephen McGregor, Mariano Mora McGinity, Sascha Griffiths

\section{FOURTH INTERNATIONAL SYMPOSIUM ON “NEW FRONTIERS IN HUMAN-ROBOT INTERACTION"}

Introduction Maha Salem, Astrid Weiss, Paul Baxter, Kerstin Dautenhahn

General Republics' Opinions on Robot Ethics: Comparison between Japan, the USA, Germany, and France ..... Tatsuya Nomura

Differences on Social Acceptance of Humanoid Robots between Japan and the UK. 258 Tatsuya Nomura, Dag Sverre Syrdal, Kerstin Dautenhahn

Presence of Life-Like Robot Expressions Influences Children's Enjoyment of Human-Robot

Interactions in the Field

David Cameron, Samuel Fernando, Emily Collins, Abigail Millings, Roger Moore, Amanda Sharkey, Vanessa Evers, Tony Prescott

The Paro Robot Seal As a Social Mediator for Healthy Users Natalie Wood, Amanda Sharkey, Gail Mountain, Abigail Millings

Can Less be More? The Impact of Robot Social Behaviour on Human Learning James Kennedy, Paul Baxter, Tony Belpaeme

Robots Guiding Small Groups: The Effect of Appearance Change on the User Experience Michiel Joosse, Robin Knuppe, Geert Pingen, Rutger Varkevisser, Josip Vukoja, Manja Lohse, Vanessa Evers

How Can a Tour Guide Robot's Orientation Influence Visitors' Orientation and Formations? 282 Daphne E. Karreman, Geke D.S. Ludden, Elisabeth M.A.G. van Dijk, Vanessa Evers

Performing Facial Expression Synthesis on Robot Faces: A Real-time Software System Maryam Moosaei, Cory J. Hayes, Laurel D. Riek

Gender, More So Than Age, Modulates Positive Perceptions of Language-based Human-robot

Interactions Megan Strait, Priscilla Briggs, Matthias Scheutz

Perception of Artificial Agents and Utterance Friendliness in Dialogue. Sascha Griffiths, Friederike Eyssel, Anja Philippsen, Christian Pietsch, Sven Wachsmuth

Turn-yielding Cues in Robot-human Conversation Jef A. van Schendel, Raymond H. Cuijpers

Robot Learning from Verbal Interaction: A Brief Survey Heriberto Cuayahuitl

Embodiment, Emotion, and Chess: A System Description Christian Becker-Asano, Nicolas Riesterer, Julien Hue, Bernhard Nebel

Towards a Child-Robot Symbiotic Co-Development: A Theoretical Approach

Vicky Charisi, Daniel Davison, Frances Wijnen, Jan van der Meij, Dennis Reidsma, Tony Prescott, Wouter van Joolingen, Vanessa Evers

Does Anyone Want to Talk to Me? - Reflections on the Use of Assistance and Companion Robots in

Care Homes.

Kerstin Dautenhahn, Anne Campbell, Dag Sverre Syrdal

Robots Have Needs Too: People Adapt Their Proxemic Preferences to Improve Autonomous Robot

Recognition of Human Social Signals.

Ross Mead, Maja J. Matari

A New Biomimetic Approach Towards Educational Robotics: The Distributed Adaptive Control of a

Synthetic Tutor Assistant.

Vasiliki Vouloutsi, Maria Blancas Munoz, Klaudia Grechuta, Stephane Lallee, Armin Duff, Jordi-Ysard Puigbo

Llobet, Paul F.M.J. Verschure 
FROM MENTAL "ILLNESS" TO DISORDER AND DIVERSITY: NEW DIRECTIONS IN THE PHILOSOPHICAL AND SCIENTIFIC UNDERSTANDING OF MENTAL DISORDER

Psychiatry and the Poverty of Subjectivity: How Phenomenology Can Contribute to the Validation of Categories of Disorder. Anthony Vincent Fernandez

The Juice is in the Detail: An Affordance-based View of Talking Therapies 364 Mark McKergow

Are Mental Disorders Illnesses? the Boundary Between Psychiatry and General Medicine 371 Valentina Petrolini

An Encounter Between Attachment Theory and 4e Cognition 378 Dean Petters, Everett Waters

\section{EMBODIED COGNITION, ACTING AND PERFORMANCE}

Image Theatre and Digital Story-telling: Towards a Research Method Called 'Collaborative

Embodied Participant Analysis' (CEPA) Michael Carklin

Stanislavsky's Mindful Actor: The System as a Guide to Experiencing Embodiment .385 Ysabel Clare

Better Than Life; Testing Techniques for an Online Audience to Influence and Participate in a Live

Performance Nicky Donald, Marco Gillies

The Cognitive Dynamics of Performance Generating Systems: Deborah Hay through Christopher House Pil Hansen

Acted Emotion: A Performance Experiment in Psychology and Actor Training..... David Jackson

Enacting Desire: Constructing Social Flexibility through Somatic-informed Processes 388 Thomas Kampe

Watergait: Designing Sense Perceptions for Individual Truth Esthir Lemi, Marientina Gotsis, Vangelis Lympouridis

Participatory Enaction of Music: Key Points Towards Radicalizing the Notion of Embodiment in Music. Juan Loaiza

The Embodied Brain: An Argument from Neuroscience for Radical Embodied Cognition Julian Kiverstein, Mark Miller

Stanislavski's System and a Dual-Process Approach to Performer Training. 393 Grant Olson

Attempts on Margarita (Multiple Drafts): A Cognitive Dramaturgy Generated by Voice and Space Christina Penna

Extended Body in the Telematics Performance: The Perceptual System of Remote Dancers Ivani Santana

The Pleasure of Not Finding Things Out: Dramaturging with Boundary Objects Freya Vass-Rhee

The Embodiment of Sound in an Intermedial Performance Space Caroline Wilkins

Author Index 